Frances F. Chung MD FRCPC, Allan Chung MD, Rosemarie H. Meier MB ChB MSc FRCP, Erika Lautenschlaeger MD FRCPC, Chanth Seyone MD

\title{
Comparison of perioperative mental function after general anaesthesia and spinal anaesthesia with intra- venous sedation
}

This study compared the postoperative mental function in 44 elderly patients following general anaesthesia (GA) or spinal anaesthesia (SA) with sedation for transurethral resection of prostate. The Mini-Mental State (MMS) was done preoperatively and postoperatively at six hours, one day, three days, five days and one month. The geriatric mental status examination was performed preoperatively and one month after the anaes. thetic. There was no significant intergroup difference in the MMS score in the preoperative, six hours, one day, three days, five days and 30 days postoperative scores between the $G A$ and $S A$ with sedation groups. A significant intragroup difference between preoperative and postoperative MMS score was detected in the GA group $(P<0.02)$ and in the SA group with sedation $(P<0.03)$. In the GA group, the significant decrease in MMS score occurred at $6 \mathrm{~h}$ postoperatively $(P<0.002)$ whereas in the SA group with sedation, MMS score also decreased significantly at $6 h(P<0.005)$. In conclusion, there was no significant difference in perioperative mental function between the general and spinal anaesthetic groups when supplemental IV sedation was given. In both groups, perioperative mental function decreased significantly at 6 h postoperatively.

\section{Key words}

ANAESTHETIC TECHNIQUES: balanced; spinal; ANAESTHETICS, INTRAVENOUS: droperidol, fentanyl; AGE FACTORS: COMPLICATIONS: postoperative confusion; HYPNOTICs: benzodiazepines, diazepam; droperidol.

From the Departments of Anaesthesia and Psychiatry, Toronto Westem Division, The Toronto Hospital, University of Toronto, Toronto.

Address correspondence to: Dr. F. Chung, Department of Anaesthesia, Toronto Western Hospital, 399 Bathurst Street, Toronto, Ontario, Canada M5T 2 S8.

Supported in part by a grant of the physicians of Ontario through the Physician Services Incorporated Foundation.
Postoperative impairment of mental function is well recognized, ${ }^{1,2}$ and may contribute to mortality and morbidity in the elderly. However, contradictory results concerning postoperative mental function have been reported in the elderly. ${ }^{3-5}$

Bigler and co-workers found no impairment in mental function after either general or spinal anaesthesia. ${ }^{3}$ However, Hole and co-workers found that the incidence of postoperative mental deterioration was lower in patients following epidural anaesthesia, compared with general anaesthesia. ${ }^{4}$ In addition, Riis and co-workers reported short-term impairment in elderly patients within the first postoperative week after either epidural or general anaesthesia. ${ }^{5}$

In a previous study, ${ }^{6}$ we found that mental function was better maintained following spinal anaesthesia when compared with general anaesthesia in the perioperative period and six hours after operation, but there was no difference in the first few days and one month postoperatively. The majority of patients who were given spinal anaesthesia in that study received no IV sedation, and this may have accounted for less perioperative mental deterioration in the spinal group, compared with general anaesthesia. The aim of the present study was to compare the postoperative mental function in elderly patients following general anaesthesia or spinal anaesthesia with sedation for transurethral resection of prostate.

\section{Methods}

The study was approved by our University Human Subjects Review Committee. We studied 44 patients who were scheduled for transurethral resection of prostate, and informed consent was obtained. Inclusion criteria were patients 60 years old or more, with normal mental status capable of giving informed consent, with no clinical evidence of significant cardiorespiratory problems, such as congestive heart failure or recent myocardial infarction, and with no medical contraindication to either 
general or spinal anaesthesia. Exclusion criteria were patients with senile dementia, previous history of psychiatric disorders, previous stroke, head trauma, inadequate command of English to participate in the tests, or an unwillingness to be randomized to either kind of anaes thesia. No premedication was given. The patients were randomly allocated to general or spinal anaesthesia with sedation.

General anaesthesia was induced with $3 \mathrm{mg} \mathrm{d}$ tubocurarine, thiopentone $2-5 \mathrm{mg} \cdot \mathrm{kg}^{-1}$ and fentanyl $2 \mu \mathrm{g} \cdot \mathrm{kg}^{-1}$ IV. The trachea was intubated after $1.5 \mathrm{mg} \cdot \mathrm{kg}^{-1}$ succinylcholine IV. Anaesthesia was maintained with a nitrous oxide-oxygen mixture ( $30-40$ per cent oxygen) plus isoflurane $0-0.5$ per cent as required via a semi-closed circle system using intermittent positive pressure ventilation. Patients also received vecuronium IV for muscle relaxation, with dose adjustments based on peripheral nerve stimulator-induced twitch tension or train- of-four response. Fentanyl $50-150 \mu \mathrm{g}$ was given IV for added analgesia as required. End-tidal carbon dioxide was monitored with mass spectrometry, and maintained within normal limits, $30-40 \mathrm{mmHg}$. After surgery, neuromuscular blockade was reversed with glycopyrrolate $0.01 \mathrm{mg} \cdot \mathrm{kg}^{-1}$ and neostigmine $0.05 \mathrm{mg} \cdot \mathrm{kg}^{-1} \mathrm{IV}$.

Spinal anaesthesia was administered through a 22- or 25-gauge spinal needle inserted in the midline of $\mathrm{L}_{3-4}$ or $\mathrm{L}_{4-5}$ interspace with the patient in the lateral decubitus position. A single dose of either tetracaine, $10-14 \mathrm{mg}$ diluted in ten per cent dextrose solution, or xylocaine 50-90 mg, was injected into the subarachnoid space. If the decrease in systolic arterial blood pressure exceeded 30 per cent of the preoperative value, in spite of IV crystalloid administration, IV ephedrine was given in 5 to $10 \mathrm{mg}$ boluses. Supplemental IV sedation, diazepam, droperidol, or fentanyl was given by the individual anaesthetist as needed for patient relaxation. No attempt was made to control the amount of IV sedation given.

In the postoperative period, meperidine $50-75 \mathrm{mg} \mathrm{IM}$ every 4-6 hr as needed was given for $24-48 \mathrm{hr}$, followed by acetaminophen with $30 \mathrm{mg}$ codeine every $4-6 \mathrm{hr}$ as required. An antiemetic, dimenhydrinate $50 \mathrm{mg}$ IM every 4-6 hr, was given as needed for nausea and vomiting. If hypnotics were required, haloperidol $0.5-1 \mathrm{mg}$ was given.

Haemoglobin, blood sugar, and serum electrolyte concentrations, analysis of arterial blood gases, chest $x$-ray and electrocardiogram were measured preoperatively. These tests, as well as liver and renal function tests, were repeated on the first day after surgery.

All psychological measures were made by two investigators who were unaware of the type of anaesthetic given. First, the Mini-Mental State (MMS), as shown in the appendix, was performed. This cognitive screening test was selected because it combines a high validity and reliability with brevity and ease of application, thereby avoiding fatigue of elderly subjects. ${ }^{7}$ This instrument lends itself to serial testing in conditions which tend to fluctuate, e.g., postoperative delirium. The MMS consists of 11 questions probing for orientation to time and place, registration, attention, calculation, short-term recall, language ability, and constructional ability (BenderGestalt design). The maximum score is 30 points, with 23 being the cutoff for cognitive impairment. The test was given preoperatively and postoperatively at six hours, one day, three days, five days, and one month. Secondly, the Geriatric Mental Status Examination (GEMS) was performed. The GEMS has been developed for use in diagnostic studies in the elderly. ${ }^{8}$ This standardized clinical interview schedule inquired into physical health; anxiety and tension; level of energy; somatic complaints; depressive and hypomanic mood; thought and perceptual disorder; and alcohol and drug abuse. It provided a record of ratings and a potential score. This was done preoperatively and one month after the anaesthetic.

Each anaesthetic group was examined by age, duration of operation, intraoperative and postoperative medication. The profiles on MMS were examined for individual patients. Statistical significance between the two groups was analyzed by the two-sided Student's $t$ test, an analysis of variance, or the Chi-square test. Change in MMS score in the GA or SA group in the postoperative period was analyzed by repeated measures analysis of variance, with contrast at each specific interval.

\section{Results}

Forty-four patients undergoing transurethral resection of prostate participated in the study. Of the 44 patients, half of the patients had general anaesthesia (GA), and half had spinal anaesthesia (SA). There were no statistically significant differences between the GA and SA groups with respect to age, ASA physical status, duration of surgery or alcohol consumption (Table I).

TABLE I Demographic data"

\begin{tabular}{|c|c|c|}
\hline Meatt $\pm S E M$ & $\begin{array}{l}\text { General } \\
\text { anaesthesia }\end{array}$ & $\begin{array}{l}\text { Spinal } \\
\text { anaesthesia }\end{array}$ \\
\hline Age (yrs) & $71.8 \pm 1.3$ & $72.2 \pm 1.3$ \\
\hline ASA physical status & $\begin{array}{lll}\text { I } & \text { II } & \text { III } \\
10 \% & 55 \% & 35 \%\end{array}$ & $\begin{array}{lll}\text { I } & \text { II } & \text { III } \\
9.5 \% & 47.6 \% & 42.9 \%\end{array}$ \\
\hline Weight (kg) & $72.1 \pm 2.0$ & $71.0 \pm 2.4$ \\
\hline Height $(\mathrm{cm})$ & $166.8 \pm 1.4$ & $170.4 \pm 1.8$ \\
\hline Duration of surgery (min) & $72.5 \pm 3.8$ & $68.0 \pm 3.4$ \\
\hline $\begin{array}{l}\text { Alcohol consumption } \\
1-2 \text { drinks } \cdot \text { day }^{-1}(\%)\end{array}$ & 25.6 & 30.5 \\
\hline
\end{tabular}

*No significant difference between groups. 
TABLE II Intraoperative data (mean \pm SEM)

\begin{tabular}{lll}
\hline General anaesthesia & \multicolumn{2}{l}{ Spinal anaesthesia } \\
\hline Thiopentone & $264.2 \pm 11.7 \mathrm{mg}$ & Tetracaine $10.8 \pm 0.3 \mathrm{mg} \mathrm{n}=4$ \\
Succinylcholine $109.1 \pm 3.8 \mathrm{mg}$ & Xylocaine $73.6 \pm 3.2 \mathrm{mg} \mathrm{n}=18$ \\
Fentanyl & $97.2 \pm 5.5 \mu \mathrm{g}$ & \\
& & Intraoperative sedation \\
& Fentanyl $50.0 \pm 7.4 \mu \mathrm{g} \mathrm{n}=10$ \\
& Diazepan $5.8 \pm 2.2 \mathrm{mg} \mathrm{n}=3$ \\
& Fentanyl $46.4 \pm 3.6 \mu \mathrm{g} ; \mathrm{n}=7$ \\
& Diazepam $3.0 \pm 0.3 \mathrm{mg}$ \\
& Fentanyl $50.0 \pm 0 \mu \mathrm{g} ; \mathrm{n}=2$ \\
& Droperidol $1.5 \pm 1.0 \mathrm{mg}$ \\
\hline
\end{tabular}

The amount of general anaesthesia required is shown in Table II. In the SA group with sedation, ten patients received IV fentanyl, three received IV diazepam, seven received a combination of IV fentanyl and diazepam, and two both IV fentanyl and droperidol. The dosage of supplemental IV sedation is shown in Table II. There was no significant difference between the GA and SA groups with respect to postoperative use of analgesic, antiemetic or sedative,

The major surgical complications were difficulty with blood clots in the bladder, bleeding and postoperative urinary retention in six patients: four received GA, and two had SA. One patient in the GA group required re-intubation due to inadequate relaxant reversal.

In the GA group, haemoglobin, sodium, potassium and blood urea nitrogen concentrations showed significant decreases postoperatively, while only the haemoglobin, sodium, and blood urea nitrogen concentrations showed a significant decrease in the SA group (Table III). Blood sugar and arterial blood gas analysis showed no significant changes from the preoperative values. There were no statistically significant differences in laboratory investigations between the groups.

There was no significant intergroup difference in the MMS score in the preoperative, six hours, one day, three days, five days and 30 days postoperative scores between the GA and SA group with sedation. A significant intragroup difference between preoperative and postoperative MMS score was detected in the GA group $(P<0.02)$ and in the SA group with sedation $(\mathrm{P}<0.03$ ) (MAN-

TABLE II] Mean decrease in laboratory values

\begin{tabular}{llc}
\hline Mean \pm SEM & \multicolumn{2}{l}{ General andesthesia Spinal anaesthesia } \\
\hline Haemoglobin $\left(\mathrm{g} \cdot \mathrm{dl}^{-1}\right)$ & $1.0 \pm 0.2^{*}$ & $0.8 \pm 0.2^{*}$ \\
Sodium $\left(\mathrm{mmol} \cdot \mathrm{L}^{-1}\right)$ & $2.0 \pm 0.5^{*}$ & $2.2 \pm 0.8^{*}$ \\
$\begin{array}{l}\text { Potassium }\left(\mathrm{mmol} \cdot \mathrm{L}^{-1}\right) \\
\begin{array}{l}\text { Blood urea nitrogen } \\
\left(\mathrm{mmol} \cdot \mathrm{L}^{-1}\right)\end{array}\end{array}$ & $0.1 \pm 0.1^{*}$ & $0.03 \pm 0.4$ \\
\hline
\end{tabular}

*P $<0.05$. Pastoperative day 1 data significantly changed from preoperative data.

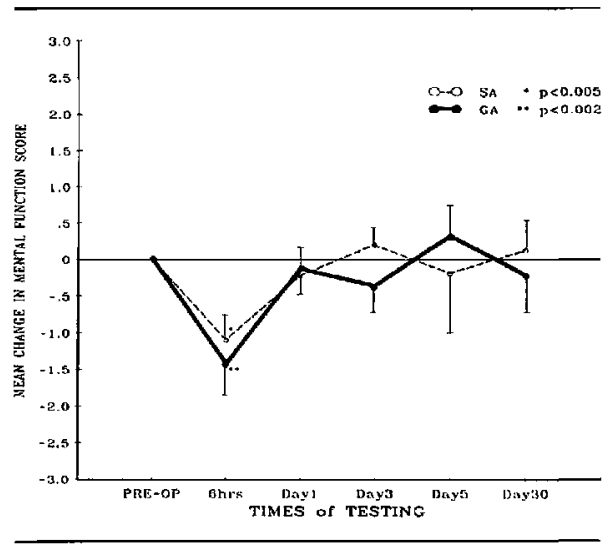

FlGURE Mean change in Mini-Mental State score for general anaesthetic and spinal anaesthetic groups, ${ }^{*}(\mathrm{P}<0.005)$ indicates significant differences compared with the preoperative scores within SA group, ${ }^{* *}(\mathrm{P}<0.002)$ indicates significant differences compared with preoperative scores within GA group.

OVA, repeated measures). In the GA group, the significant decrease in MMS score occurred at $6 \mathrm{hr}$ postoperatively ( $P<0.002$ ), and in the SA group with sedation, MMS score also decreased significantly at $6 \mathrm{~h}(\mathrm{P}<$ 0.005 ). No statistically significant difference in the postoperative MMS score was found following the different types of IV sedation in the SA group. There were no significant differences in the amounts of postoperative analgesic, antiemetic or hypnotics between the GA and the SA groups.

If the patient's score on the MMS examination decreased two or more points, they met the clinical criterion for cognitive decline. ${ }^{9}$ Of 22 patients with $G A$, ten had a decline in their MMS score of two or more points, and eight out of 22 patients in the SA group with sedation had a decreased score of two or more perioperatively (GA 45.5 per cent vs SA 36.4 per cent NS).

The GEMS examination showed that all patients were of normal mental status. There was no difference between the GA and SA groups preoperatively and postoperatively.

Two patients out of 44 patients were confused ( 4.5 per cent). Each had received $G A$, representing an incidence of nine per cent for this group. There was no significant difference in incidence of postoperative confusion between patients receiving GA or SA. The first confused patient was an anxious 77-year-old who developed postoperative bladder spasm requiring treatment with anticholinergic drugs. He developed confusion on the third postoperative day. The second was a 64 -year-old 
who developed confusion on the first operative day. He had a history of angina and was being treated with multiple dnug therapy for arrhythmia and atherosclerotic heart disease.

\section{Discussion}

In our previous study, we found a significant impairment of cognitive function at six hours postoperatively, and some impairment at three days postoperatively following GA. ${ }^{6}$ The maintenance of mental function was better following SA, when compared with GA.

Contrary to our previous findings, this study indicated that there was no significant difference in postoperative mental function between the GA and SA groups when intraoperative sedation was given. Mental function decreased significantly in the general anaesthetic group at six hours postoperatively. Also, in the SA group with sedation, mental function decreased significantly at six hours postoperatively.

This difference in results could be due to differences in intraoperative sedation during SA. In our previous study, the majority of patients received verbal reassurance during $\mathbf{S A}$, and minimal intraoperative sedation was given. In this study, the $\mathrm{SA}$ group received supplemental IV sedation. Their postoperative mental function score decreased significantly. Although no statistically significant difference in the postoperative MMS score was found between the different types of IV sedation, as little as 2.5 mg diazepam has been shown to decrease mental function and slow psychomotor performance in the elderly. ${ }^{10}$

Many patients fear being awake in the operating room. The acceptance of SA is often made dependent upon the promise of adequate sedation. With increasing age, patients become more sensitive to sedative and hypnotic drugs, and recover more slowly from their effects. In the elderly, the cardiorespiratory depressing effects of these drugs can potentially accentuate cerebral anoxia. The resulting agitation and delirium may result in more sedation being given which perpetuates a vicious circle.

Caplan analyzed 14 cases of sudden cardiac arrest in healthy patients who received SA. One of the common patterns in these cases was the intraoperative use of sufficient sedation to produce an apparently comfortable, sleep-like state which could have resulted in unrecognized respiratory insufficiency that might have contributed to the cardiac arrest. " Thus use of supplemental IV sedation during regional anaesthesia may not be as harmless as is generally accepted.

Intraoperatively, emotional support of the elderly patient by verbal reassurance is often ignored because it is easier to give IV sedation. The anaesthetist is in the unique position of being able to help by giving support by psychological rather than pharmacological means. This study demonstrates the decrease in mental function after SA with IV sedation. The cardiorespiratory depressing effects of these drugs are known and should make us re-examine the practice of routine IV sedation during regional anaesthesia. If IV sedation is considered to be necessary, we should use shorter-acting agents so that their effects will not persist in the recovery period.

Mini-Mental State was chosen as a clinical instrument to screen and assess cognitive function. It is clinically acceptable because it is easy to administer and complete when fatigue and inattention occur in elderly patients after surgery. There was no difference in postoperative mental function between patients receiving $S A$ and $G A$. Using a sample size of 44 patients, the power of this study to detect a specified between group difference is 80 per cent. Although the absence of a significant difference in the two groups does not exclude the possibility that more sophisticated tests might have defined the presence of a difference, there was no clinically feasible instrument which could have been applied in the elderly.

Similar to Berggren et al.,$^{12}$ we found no difference in the incidence of confusion between the two anaesthetic techniques. Two patients in the GA group developed postoperative confusion, one requiring anticholinergic drugs for bladder spasm and the other multiple drug therapy for heart disease.

Our previous study also showed that two patients developed postoperative confusion: each was receiving tricyclic antidepressants. Berggren et al. found 44 per cent of their patients developed confusion, which correlated closely to a history of mental depression and to the use of drugs with anticholinergic effects. ${ }^{12}$ Thus drug therapy, especially anticholinergic, rather than the anaesthetic technique, appears to have a greater role in postoperative confusion.

Serum levels of anticholinergic drugs are important in the development of confusional states. There is an important relationship between cognitive impairment and serum levels of anticholinergics in patients who have experienced delirium after cardiotomy. ${ }^{13}$ Although many of the anaesthetic drugs are not considered to be anticholinergic agents, they may block muscarinic receptors directly or via their metabolites. ${ }^{14}$

With the development of less irritant inhalational anaesthetic agents, the routine preoperative administration of anticholinergics could be abolished. If anticholinergic agents are required perioperatively, synthetic anticholinergic agents with quaternary ammonium derivative, e.g., glycopyrrolate, should be used instead of atropine or hyoscine. The quaternary ammonium compounds do not cross the blood-brain barrier because of their net positive charge, and rarely produce central nervous system toxicity. ${ }^{15}$ 
Most postoperative delirious states are attributable to toxic or metabolic insults, including dehydration, anaemia, hypoxia, acid-base imbalance, hypocarbia, hypercarbia, hypoglycaemia, hyperosmolarity, hypotension, and endocrinopathies. ${ }^{16}$ We did not find any significant degree of hypoxaemia preoperatively or one day postoperatively in either general anaesthesia or spinal anaesthesia group. This may be due to rapid mobilization of patients after transurethral resection of prostate. However, there was a significant decrease in haemoglobin, sodium, and blood urea nitrogen concentrations in patients who had either GA or SA, and these changes could be contributing factors to postoperative confusion.

In summary, in this study we have demonstrated that there was no significant difference in postoperative mental function between the GA and SA groups when supplemental IV sedation was given. In both groups, perioperative mental function decreased significantly at six hours postoperatively. The elderly are more sensitive to sedatives and analgesic drugs, and in addition, they may also have underlying organic brain disease including Parkinsonism or a previous cerebrovascular accident. Thus, potential dangers exist for them to become disoriented in the perioperative period. What is considered to be a normal dose of sedation may be a relative overdose in the elderly, and can precipitate cardiorespiratory depression and mental confusion. Thus, we should re-examine the practice of giving supplemental IV sedation during SA. The benefits of sedation, less anxiety and more comfort, should be balanced against the potential deleterious effects of sedative drugs such as transient cognitive impairment and respiratory insufficiency. If IV sedation is needed, shorter-acting agents in minimal amounts should be given together with verbal reassurance during spinal anaesthesia.

\section{Acknowledgments}

The authors thank Miss C. Drane for her secretarial assistance, Mr. A. Ayiomamitis for statistical analysis, and Dr. J. M. R. Campbell for his support in this research.

\section{References}

1 Simpson BR, Williams $M$, Scott $J F$ et al. The effects of anaesthesia and elective surgery on old people. Lancet 1961; 2: 887-93.

2 Blundell $E$. A psychological study of the effects of surgery on eighty-six elderly patients. Br J Soc Psychol 1967; 6: 297-303.

3 Bigler $D$, Adelhoj $B$, Petring $O U$ et al. Mental function and morbidity after acute hip surgery during spinal and general anaesthesia. Anaesthesia 1985; 40; 672-6.
4 Hole A, Terjesen T, Brievik H. Epidural versus general anesthesia for total hip arthroplasty in elderly patients. Acta Anaesthesiol Scand 1980; 24: 279-87.

5 Riis $J$, Lomholt $B$, Haxholdt $O$ et al. Immediate and long term recovery from general versus epidural anaesthesia in elderly patients. Acta Anaesthesiol Scand 1983; 27: 44-9.

6 Chung $F$, Meier $R$, Lautenschiager $E$ et al. General or spinal anesthesia: which is better in the elderly? Anesthesiology 1987; 67: 422-7.

7 Folstein M, Folstein S, McHugh P. "Mini Mental State." A practical method for grading the cognitive state of patients for the clinician. J Psychiatr Res 1975; 12: 189-98.

8 Duckworth GS. The reliability of GEMS. Proceedings of the Ontario Psychogeriatric Association, September, 1976; 54-9.

9 Mondimore FM, Damlouji N, Folstein MF, Tune L. PostECT confusional states associated with elevated serum anticholinergic levels. Am J Psychiatry 1983; 140: 930-1.

10 Pomara N, Stanley $B$, Block $R$ et al. Increased sensitivity of the elderly to the central depressant effect of diazepam. J Clin Psychiatry 1985; 46: 185-7.

11 Caplan RA, Ward RJ, Posner K, Cheney FW. Unexpected cardic arrest during spinal anesthesia: a close claims analysis of predisposing factors. Anesthesiology 1988; 68: 5-11.

12 Berggren D, Gustafon Y, Eriksson B et al. Postoperative confusion after anesthesia in elderly patients with femoral neck fractures. Anesth Analg 1987; 66: 497-504.

13 Tune LE, Damiouji NF, Holland A et al. Association of postoperative delirium with raised serum levels of anticholinergic drugs. Lancet 1981; 1: 651-2.

14 Summers WK. A clinical method of estimating risk of drug induced delirium. Life Sci 1978; 22: 1511-6.

15 Greenblatt $D J$. Shader $R I$. Drug therapy: anticholinergics. N Engl J Med 1975; 288: 1215-8.

16 Lerkoff SE. Besdine RW, Wette T. Chapter: Acute confusional states in the hospitalized elderly. In: Eisdorfer C (Ed.). Annual Review of Gerontology and Geriatrics Volume 6. New York: Springer, 1986; 1-26.

\section{Résumé}

Dans ce travail, nous comparons les fonctions mentales de 44 patients agés, après une résection trans-urétrale de la prostate sous anesthésie soit générale $(A G)$ soit rachidienne avec sédation (ARS). Nous avons fait un examen mental gériatrique (GEMS) avant et un mois après l'opération ainsi qu' un examen plus sommaire, le Mini-Mental State (MMS) juste avant et 6, 24, 72, et 120 heures de même qu' un mois après l' intervention. Avec le MMS, nous n'avons pas identifié de différence significative 
entre les deux groupes à quelque moment que ce soit. A l'intérieur de chacun des deux groupes cependant, on a noté une baisse des résultats au MMS entre le pré-el le post-opératoire $(P$ $<0.02$ pour $A G$ et $P<0.03$ pour ARS) qui était détectable six heures après l'intervention ( $P<0.002$ pour $A G$ et $P<0.005$ pour ARS). L'état mental post-opératoire des patients n'était donc pas dépendant du type d'anesthésie, qu' elle soit générale ou rachidienne avec sédatifs par voie intraveineuse.

\section{Appendix (Mini-Mental State)}

\section{Score Score} (day) (month)?

5 ( ) Where are we: (state) (county) (town) (hospital) (floor)?

\section{Registration}

( ) Name 3 objects: 1 second to say each. Then ask the patient all 3 after you have said them. Give 1 point for each correct answer. Then repeat them until he learns all 3. Count trials and record. Trials

Attention and calculation

( ) Serial 7's. 1 point for each correct. Stop after $\mathbf{5}$ answers. Alternatively spell "world" backwards.

\section{Recall}

( ) Ask for the 3 objects repeated above. Give 1 point for each correct.

\section{Language}

( ) Name a pencil, and watch (2 points) Repeat the following "No ifs, ands or buts." (1 point)

Follow a 3-stage command:

"Take a paper in your right hand, fold it in half, and put it on the floor." ( 3 points)

Read and obey the following:

Close your eyes (1 point) Write a sentence (1 point) Copy design (1 point)

\section{( ) Total score}

